\title{
Acompanhamento farmacoterapêutico em pacientes dislipidêmicos de um lar de idosos da cidade de Novo Hamburgo-RS
}

\author{
The pharmacotherapeutic follow-up in dyslipidemic patients of a nursing home in Novo Hamburgo \\ city, state of Rio Grande do Sul, Brazil
}

Fransuelen de Gregori ${ }^{1}$

Ana Luiza Ziulkoski

Letícia Hoerbe Andrighetti

Eloir Dutra Lourenço2

Magda Susana Perassolo?

\section{Resumo}

Introducão: A partir do último século, observou-se aumento na expectativa de vida e na incidência de patologia na população idosa, principalmente doenças crônicas. Objetivo: Avaliar o efeito do acompanhamento farmacoterapêutico (AF) em pacientes dislipidêmicos de um lar de idosos da cidade de Novo Hamburgo, RS. Metodologia: Trata-se de estudo quantitativo, observacional com delineamento longitudinal retrospectivo, que avaliou 50 pacientes idosos residentes de um lar geriátrico $(80,2$ \pm 7,64 anos, 32 mulheres). Foi avaliado o perfil lipídico desses pacientes (colesterol total, triglicerídeos, HDL e LDL) antes e após AF de um ano. A análise dos resultados ocorreu por meio de estatística descritiva e teste $t$ Student ou U de Mann Whitney para amostras pareadas. Resultados: $56 \%$ dos pacientes apresentaram alteração no perfil lipídico no início do estudo e, $30 \%$ ao final, ocorrendo significativa melhora após o AF. Além disso, os níveis de colesterol total apresentaram diminuição favorável após um ano de acompanhamento (206 \pm 53 vs. $180 \pm 43 \mathrm{mg} / \mathrm{dL} ; \mathrm{P}=0,009)$. A maioria dos pacientes que apresentou diagnóstico de dislipidemia utilizava medicamentos há pelo menos três meses para o tratamento dessa patologia (estatinas e fibratos). A maioria desses pacientes utilizava os medicamentos de forma correta. Conclusão: Conclui-se que os pacientes tiveram melhora no seu perfil lipídico após um ano de acompanhamento.

\section{Abstract}

Introduction: Since the last century, life expectancy and the incidence of disease in the elderly have increased, especially chronic diseases. Objective: This study aims to evaluate the pharmacotherapeutic follow-up(PF) in dyslipidemic patients of a nursing home in Novo Hamburgo city, state of Rio Grande do Sul, Brazil. Methodology: This is a quantitative, observational study with a longitudinal retrospective design, evaluating 50 elderly patients who live in a nursing home (80.2 \pm 7.64 years old, 32 women). We assessed the lipid profile of these patients (total cholesterol, triglycerides, HDL and LDL) before and after one-year PF. The analysis was conducted through descriptive
Palavras-chave:

Idoso. Dislipidemias. Uso de Medicamentos. Acompanhamento Farmacoterapêutico.

Key words: Elderly. Dyslipidemias. Drug Utilization. Pharmaceutical Monitoring.

\footnotetext{
Curso de Farmácia, Instituto de Ciências da Saúde. Universidade Feevale. Novo Hamburgo, RS, Brasil.

2 Curso de Biomedicina, Instituto de Ciências da Saúde. Universidade Feevale. Novo Hamburgo, RS, Brasil.
} 
statistics and Student $t$ test or U of Mann Whitney test for paired samples. Results: $56 \%$ of the patients presented changes in lipid profile in the beginning of the study and $30 \%$ one year later, with significant improvement of the lipid profile after the monitoring. Moreover, total cholesterol levels showed a favorable decrease after a year of monitoring (206 \pm 53 vs. $180 \pm 43 \mathrm{mg} / \mathrm{dL} ; \mathrm{P}=0.009)$. Most patients diagnosed with dyslipidemia were using drugs, for at least three months, to treat this pathology (statins and fibrates). The majority of these patients used them correctly. Conclusion: The patients had significant improvement in their lipid profile after one year of monitoring.

\section{INTRODUÇÃO}

No decorrer do último século, observouse um aumento da longevidade da população, sendo que o envelhecimento demográfico é uma tendência estabelecida mundialmente. ${ }^{1} \mathrm{O}$ envelhecimento da população brasileira teve início a partir da década de 1960, com o declínio sustentado da fecundidade e manutenção da queda da mortalidade, resultando em um processo contínuo de estreitamento da pirâmide etária. ${ }^{2}$ Assim, a expectativa de vida do ser humano aumentou e, consequentemente, maior é a incidência de doenças e problemas associados aos idosos. ${ }^{3}$

A transição de uma população jovem para uma envelhecida vem sendo acompanhada por modificações no perfil epidemiológico da população brasileira. As doenças infectocontagiosas, altamente prevalentes em populações jovens, tendem a diminuir sua incidência, enquanto as doenças crônicas nãotransmissíveis aumentam sua prevalência. ${ }^{4}$ Além disso, estudos epidemiológicos demonstram um aumento na prevalência de doenças, com o envelhecimento, originadas por uma nutrição inapropriada e por um estilo de vida sedentário. Ao mesmo tempo, o envelhecimento é o maior fator de risco para doenças crônicas, especialmente as cardiovasculares (DCV) ou arteriocoronarianas (DAC), muitas vezes resultantes de alterações no perfil lipídico do idoso. ${ }^{5}$ Consequentemente, também ocorre um aumento do consumo de medicamentos por essa população, tornando-as a faixa etária que mais os utiliza. ${ }^{5}$
Define-se dislipidemia como um quadro clínico caracterizado por concentrações anormais de lipídios ou lipoproteínas no sangue, sendo um dos principais fatores que determinam o desenvolvimento de DCV. Altas concentrações de triglicerídeos (TG), colesterol total (CT), e de sua fração LDL-colesterol (LDL), coligadas à diminuição nos valores de HDL-colesterol (HDL), elevam a probabilidade de ocorrer essas enfermidades. ${ }^{6}$

A idade, o envelhecimento dos órgãos e o surgimento de doenças concomitantes fazem do paciente idoso alvo preferencial para o aumento das taxas do perfil lipídico. A alimentação mal orientada e muitas vezes monótona, o sedentarismo, com pouca ou nenhuma atividade física, o estresse crônico formador de ansiedades, a fome compulsiva e a desmotivação são fatores $\operatorname{aditivos}^{7}$ para o surgimento das dislipidemias. Assim, a idade é uma variável preditora da terapia medicamentosa de forma geral e seu efeito se produz mesmo antes dos 60 anos, pois a chance de usar algum tipo de fármaco aumenta desde a quarta década de vida.

O aumento do uso de medicamentos após os 40 anos ocorre devido à incidência de muitas patologias que acometem esta faixa etária. Desta forma, esses indivíduos tendem a ser os maiores usuários de medicamentos. ${ }^{8,9}$ Este fato é comprovado por meio de um estudo realizado em Porto Alegre, segundo o qual 91\% dos idosos fazem uso de algum fármaco, sendo que $27 \%$ dos idosos entrevistados utilizavam cinco ou mais medicamentos. ${ }^{10}$ 
Os pacientes idosos, portanto, são os principais consumidores e os maiores beneficiários da farmacoterapia moderna. Mais de $80 \%$ tomam no mínimo um medicamento diariamente, e este é o mais poderoso processo de intervenção para melhorar o estado de saúde dos idosos. ${ }^{11,12}$ Estes achados reforçam a importância do acompanhamento farmacoterapêutico (AF) na promoção do uso correto de medicamentos. A abordagem educativa favorece o esclarecimento de dúvidas e proporciona maior efetividade na aplicação de medidas terapêuticas.

Este estudo teve como objetivo avaliar o efeito do acompanhamento farmacoterapêutico (AF) por um ano em pacientes dislipidêmicos, de um lar de idosos da cidade de Novo Hamburgo, RS.

\section{METODOLOGIA}

\section{Delineamento do estudo}

Trata-se de estudo quantitativo, observacional com delineamento longitudinal, realizado no lar de idosos São Vicente de Paula localizado no município de Novo Hamburgo, RS, em parceria com o projeto de extensão "Atenção Farmacêutica na Comunidade", vinculado à Universidade Feevale. Os participantes do estudo foram os 50 pacientes idosos residentes que são atendidos pelo referido projeto de extensão e que realizaram exames de perfil lipídico por meio do projeto "Ações Biomédicas na Comunidade" da mesma universidade.

\section{Aspectos éticos}

O projeto seguiu todos os aspectos éticos, sendo aprovado pelo Cômite de Ética em Pesquisa (CEP) da Universidade Feevale, sob o número de protocolo 4.03.03.09.1528. Todos os indivíduos que aceitaram participar do estudo assinaram o Termo de Consentimento Livre e Esclarecido (TCLE), assinado em duas vias, onde uma permaneceu com o participante e a outra com o investigador. Foram assinados dois tipos de TCLE: um destinado ao paciente (quando este foi entrevistado) e outro ao cuidador (quando o entrevistado foi o cuidador responsável pelo idoso). Este segundo termo de consentimento foi aplicado nos casos onde o paciente tinha dificuldades em esclarecer as dúvidas.

\section{Descrição da amostra}

Foram incluídos indivíduos com 60 anos ou mais, que apresentaram diagnóstico de dislipidemia e/ou que estavam em tratamento medicamentoso com hipolipemiantes no mínimo por três meses no momento do estudo. Foram excluídos pacientes que não tinham dados de exames laboratoriais para o diagnóstico de dislipidemia. Os pacientes que participaram do estudo foram acompanhados pelo período de um ano - 2009/2010 - no qual os mesmos foram avaliados no início e no final do período. A amostra analisada foi eleita por conveniência, uma vez que foram selecionados pacientes moradores de um lar geriátrico que apresentavam alteração do perfil lipídico. Isto pode ser uma das limitações do estudo em questão.

\section{Coleta de dados}

As informações relativas à pesquisa foram coletadas nos prontuários médicos dos pacientes, caderno de plantão da enfermagem e nas fichas de medicamentos. Também foram avaliados, nas planilhas do projeto de extensão "Atenção Farmacêutica na Comunidade", dados das intervenções farmacêuticas.

Quando necessário, foram realizadas conversas com o próprio paciente e/ou cuidador, a fim de obter mais informações relacionadas aos problemas com a administração dos medicamentos e possíveis efeitos adversos. Essa conversa foi realizada de maneira informal, possibilitando total liberdade dos entrevistados, uma vez que são pessoas idosas. 
Dos prontuários médicos foram verificadas informações sobre sexo, idade, patologias dos pacientes, comorbidades e observações adicionais (comportamento, sintomas, queixas, evolução de algum quadro clínico). Nas fichas de medicamentos, foram coletados dados referentes aos medicamentos, como nome genérico e/ou comercial, dosagem, horário de administração e indicação do medicamento. Os medicamentos foram classificados seguindo a classificação da ATC (Anatomical Therapeutic Chemical Classification). ${ }^{13}$

Os dados para análise dos exames laboratoriais (resultados dos exames de CT, TG, LDL e HDL) e os demais dados referentes às intervenções farmacêuticas foram coletados das planilhas disponibilizadas pelo projeto de extensão "Atenção Farmacêutica na Comunidade", por meio de uma parceria para obtenção destes dados. Buscou-se verificar quantas intervenções farmacêuticas foram realizadas, qual o profissional envolvido (médico e/ou técnico de enfermagem), o tipo de intervenção (escrita e/ ou verbal), o desfecho do caso (aceita/não aceita) e quando aceitas, qual a alteração realizada e o impacto disto para o tratamento do paciente.

\section{Determinação do perfil lipídico}

$\mathrm{O}$ método utilizado para dosagem de CT e TG foi o colorimétrico (Enzimático de Trinder), utilizando-se kit comercial da Wiener Lab e Labtest, respectivamente. A determinação de HDL foi realizada por meio de precipitação com ácido fosfotúngstico e cloreto de magnésio, seguida por método colorimétrico, usando-se kit comercial da Wierner Lab. Para a determinação do LDL, utilizou-se a equação de Friedewald, que é expressa como LDL $=\mathrm{CT}-\mathrm{HDL}-\mathrm{TG} / 5$, porém o requisito básico para a aplicação da equação é que a concentração de TG seja inferior a $400 \mathrm{mg} / \mathrm{dL} .^{14}$

Os valores de referência utilizados para a avaliação dos pacientes foram os seguintes: CT níveis inferiores a $200 \mathrm{mg} / \mathrm{dL}$; TG valores até $150 \mathrm{mg}$ / dL; LDL níveis inferiores $160 \mathrm{mg} / \mathrm{dL}$; e HDL em homens valores superiores a $40 \mathrm{mg} / \mathrm{dL}$ e mulheres valores superiores a $50 \mathrm{mg} / \mathrm{dL} .{ }^{5}$ Os parâmetros laboratoriais que se encontravam fora dos valores de referência foram considerados alterados.

\section{Análise estatística}

Os resultados foram analisados por meio de estatística descritiva (percentual, média, desviopadrão, mediana e variação). Para a análise da evolução da dislipidemia, foi utilizado o teste $t$ Student para amostras pareadas nas variáveis paramétricas e o teste $\mathrm{U}$ de Wilcoxon para as nãoparamétricas. O nível de significância adotado foi de $5 \%$. O programa estatístico utilizado foi o SPSS 15.0.

\section{RESULTADOS}

Foram avaliados 50 pacientes, dos quais $56 \%$ apresentaram alteração no perfil lipídico no início do estudo e $30 \%$ ao final, ocorrendo significativa melhora após um ano de acompanhamento. A idade média foi de 80,2 \pm 7,64 anos, sendo o sexo feminino $(64 \%)$ o predominante na amostra.

As classes de medicamentos mais utilizadas pelos pacientes foram os antipsicóticos e antihipertensivos (ambos com 19,3\%), bem como antilipêmicos $(13,3 \%)$ e diuréticos $(10,2 \%)$, seguidas por outras classes de medicamentos, conforme a tabela 1. Todos os pacientes avaliados utilizavam dois ou mais medicamentos concomitantemente. 
Tabela 1 - Classes de medicamentos (classificação ATC) utilizadas pelos residentes no Lar São Vicente de Paula. Novo Hamburgo, RS, 2009-2010.

\begin{tabular}{cc}
\hline Classes & Pacientes (\%) \\
\hline Antipsicóticos e anti-hipertensivos & 19,3 \\
Antilipêmicos & 13,3 \\
Diuréticos & 10,2 \\
Antiepilépticos & 9,0 \\
Analgésicos/Antipiréticos e antissecretores & 8,5 \\
Antianêmicos & 6,0 \\
Antidiabéticos & 5,4 \\
Dilatadores dos vasos coronarianos & 5,4 \\
Sedativos ansiolíticos & 4,9 \\
Outros* & 18,0 \\
\hline
\end{tabular}

*Analépticos; antiasmáticos; anticolinérgicos; anti-histamínicos; antimetabólitos; antiparkinsonianos; coagulação sanguínea e homostípticos; corticóides; fornecedores de água e sais minerais; insuficiência cardíaca congestiva; miorrelaxantes central; oftálmicos; quimioterápicos para tratamento respiratório e urinário; sedativos-hipnóticos; vitaminas.

Dos pacientes que apresentaram alteração no perfil lipídico no início do estudo (28), 36\% utilizavam medicamentos para controle da dislipidemia. Os demais pacientes (74\%) não utilizavam medicamentos para dislipidemia. Os antilipêmicos mais utilizados no início do estudo, com suas respectivas doses, foram sinvastatina $20 \mathrm{mg}(55,5 \%)$, genfibrozila $600 \mathrm{mg}$ $(27,8 \%)$, ciprofibrato $100 \mathrm{mg}$ e sinvastatina 40 mg (ambos com $11,1 \%$ ) e por fim, sinvastatina $10 \mathrm{mg}(5,6 \%)$. Apenas dois pacientes utilizavam dois antilipêmicos concomitantemente. Entre os pacientes que utilizavam medicamentos antilipêmicos, $72,3 \%$ faziam administração dos medicamentos corretamente, seguindo dosagem e horário adequado. Os outros pacientes faziam uso do medicamento de forma inadequada (dosagem e/ou horário de administração incorretos). Neste caso, encontrava-se a genfibrozila $600 \mathrm{mg}$, cuja dose diária recomendada, segundo a literatura, ${ }^{15,16}$ é de $1.200 \mathrm{mg} /$ dia, dividida em duas tomadas diárias, 30 minutos antes do desjejum e do jantar. Os pacientes, porém, utilizavam $600 \mathrm{mg} / \mathrm{dia}$, somente às $20 \mathrm{~h}$, sendo administrada ainda após $\mathrm{o}$ jantar, pois este acontece às $18 \mathrm{~h}$.

Dos 36\% dos pacientes que utilizavam medicamentos antilipêmicos, apenas quatro relataram efeitos adversos relacionados ao uso destes, a saber: náuseas (1), cefaleia (2) e bulemia (1). Os demais não relataram efeitos adversos relacionados a esses medicamentos. Apenas um paciente utilizava sinvastatina e genfibrozila concomitantemente, apresentando risco de interação medicamentosa entre eles.

Comparando os resultados dos exames laboratoriais antes e após um ano de AF dos pacientes, observou-se que houve melhora nos níveis de CT (206 $\pm 53 \mathrm{mg} / \mathrm{dL}$ vs. $180 \pm 43 \mathrm{mg} /$ $\mathrm{dL} ; \mathrm{P}=0,009)$ e TG [131 (80 - 400) mg/dL e 113 $(54-442) \mathrm{mg} / \mathrm{dL} ; \mathrm{P}=0,086]$, embora este último não tenha atingido significância estatística. Os níveis de HDL diminuíram $(51 \pm 13 \mathrm{mg} / \mathrm{dL}$ vs $46 \pm 12 \mathrm{mg} / \mathrm{dL} ; \mathrm{P}=0,049)$. Os valores de LDL não sofreram alterações ao longo do estudo (122 $\pm 48 \mathrm{mg} / \mathrm{dL}$ vs. $125 \pm 44 \mathrm{mg} / \mathrm{dL} ; \mathrm{P}=0,326)$, conforme demonstrado na figura 1 . 


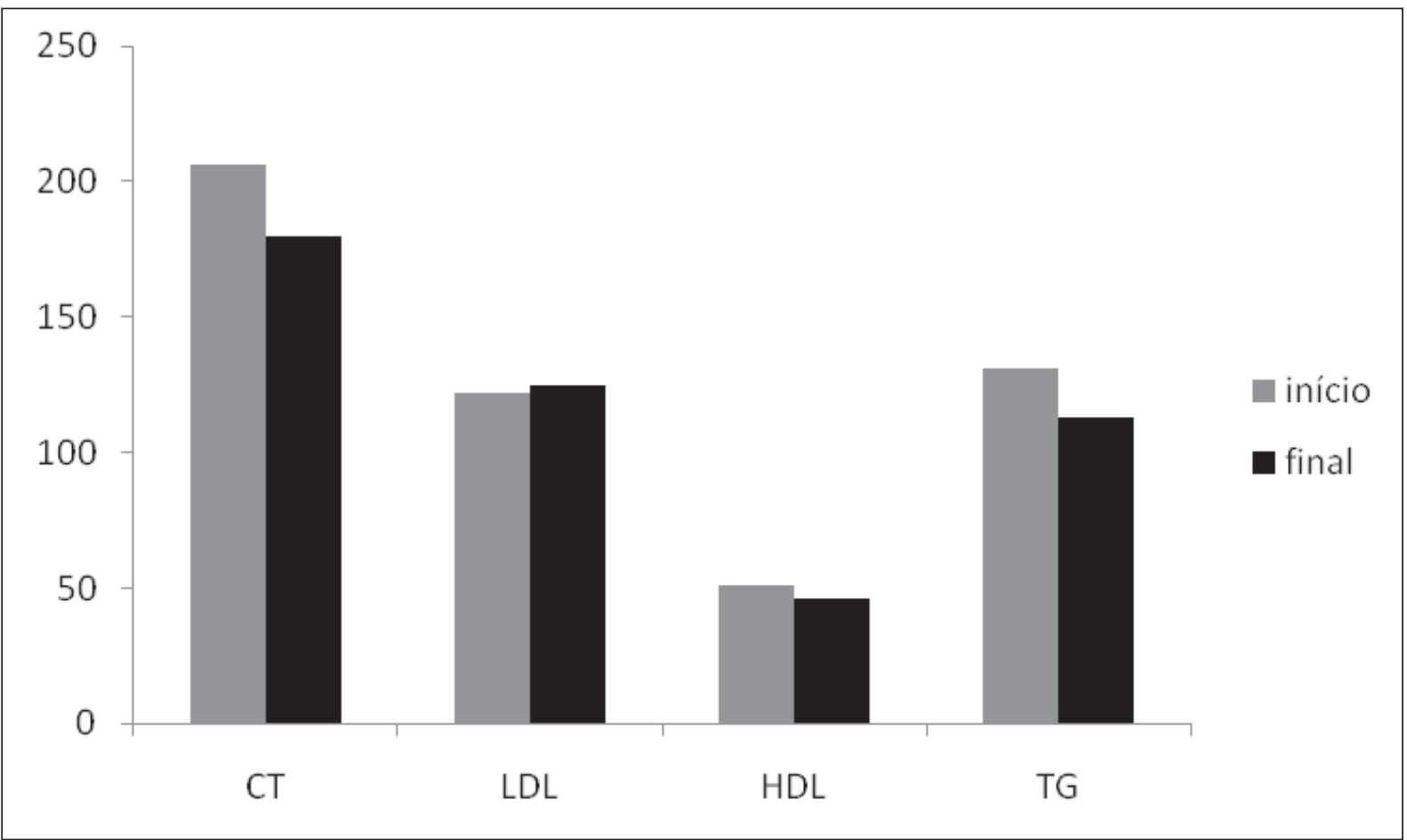

Figura 1 - Avaliação do perfil lipídico (em mg/dL) dos residentes do Lar São Vicente de Paula no início e ao final de um ano de acompanhamento. Novo Hamburgo, RS, 2009-2010.

CT: colesterol total; LDL: colesterol LDL; HDL: colesterol HDL; TG: triglicerídeos.

* $\mathrm{P}<0,05$ (teste $t$ Student para amostras pareadas; teste U de Wilcoxon - TG).

Durante o período de AF, foram realizadas 35 intervenções farmacêuticas. Destas, três foram realizadas envolvendo os medicamentos já utilizados pelos pacientes para o tratamento da dislipidemia. Todas estavam relacionadas ao medicamento genfibrozila e foram realizadas pelo farmacêutico para o técnico de enfermagem, de maneira verbal, solicitando-se adequação do horário de administração. Todas foram aceitas e ocorreu troca no horário da medicação.

Após a avaliação inicial dos pacientes, foram realizadas intervenções farmacêuticas destinadas ao médico assistente, no sentido de introduzir medicamentos para o tratamento das dislipidemias. Desta forma, foram realizadas 32 intervenções farmacêuticas, ou seja, nos pacientes que apresentavam alteração no seu perfil lipídico inicialmente. A prescrição dos medicamentos (escolha do medicamento e dose) foi realizada pelo médico que atende os moradores do local.
Dos pacientes que apresentaram alteração no perfil lipídico no início do estudo, 53\% passaram a utilizar medicamentos para o tratamento $\mathrm{da}$ dislipidemia. Ao restante desses pacientes (47\%) foram prescritas medidas dietéticas para o controle das dislipidemias. Desta forma, nem todas as intervenções propostas pelo farmacêutico, ou seja, o uso de medicação para tratamento da dislipidemia, foram aceitas pelo médico assistente.

\section{DISCUSSÃO}

No início do presente estudo, observouse alta prevalência de dislipidemia; no entanto, após uma avaliação inicial e introdução de terapia medicamentosa e controle dietético naqueles pacientes que apresentavam alteração dos níveis lipídicos, estes melhoraram após um ano de acompanhamento. Mesmo assim, 
alguns pacientes continuaram com alterações nesses parâmetros, que podem contribuir para um aumento no risco $\mathrm{CV}$ desses pacientes. ${ }^{17}$ O controle dos níveis lipídicos no organismo e, consequentemente, o desenvolvimento de doença cardíaca, é realizado com mudanças no estilo de vida, como perda de peso, realização de atividade física, mudanças dietéticas, redução do consumo de álcool e tabaco. ${ }^{18,19}$ Segundo alguns autores, essas alterações podem reduzir o LDL de $5-15 \%{ }^{20}$ e o CT em aproximadamente $14 \%{ }^{21}$

A idade média (80,2 \pm 7,64 anos) e o sexo predominante neste estudo (feminino) condizem com os dados do Estudo de Framingham, segundo o qual a hipercolesterolemia nos idosos foi mais prevalente em mulheres do que em homens e mais frequente na faixa etária dos $65-$ 74 anos e, sendo que em idades superiores a 75 anos a frequência de hipercolesterolemia tende a declinar gradativamente. ${ }^{22}$

Em relação aos medicamentos utilizados pelos pacientes que apresentaram dislipidemia, a maioria faz uso de medicamentos da classe das estatinas. Apesar dos efeitos adversos, a classe das estatinas em geral é bem tolerada e efetiva para o tratamento das dislipidemias. ${ }^{23}$ Segundo McKenney, ${ }^{20}$ o mecanismo de ação desses medicamentos consiste na inibição da enzima HMG-CoA redutase, responsável pela síntese do colesterol, e a redução de LDL ocorre pelo aumento dos seus receptores na superfície da célula hepática. A sinvastatina é o medicamento mais utilizado desta classe, uma vez que apresenta boa eficácia para redução de níveis de CT e LDL. Os outros medicamentos utilizados foram os fibratos, que são fármacos derivados do ácido fíbrico que agem estimulando os receptores nucleares denominados "receptores alfa ativados de proliferação das peroxissomas" (PPAR- $\alpha$ ). Esse estímulo leva ao aumento da produção e ação da lípase lipoproteica, responsável pela hidrólise intravascular dos TG, e também à maior síntese de Apo AI, e consequentemente de HDL. Esses fármacos sendo bastante efetivos na redução dos níveis de TG. ${ }^{15}$

Os níveis de CT apresentaram significativa redução após um ano de acompanhamento, o que indica que o tratamento farmacológico foi efetivo. Desta forma, pode-se considerar que o tratamento com a sinvastatina, medicamento mais utilizado pelos pacientes, apresentou resultados satisfatórios, sendo considerado um medicamento eficaz no tratamento dessa patologia. Também houve redução nos níveis de TG após o acompanhamento de um ano, o que indica um tratamento efetivo para esta alteração.

Os níveis de HDL diminuíram após um ano de acompanhamento, o que não é um resultado satisfatório, uma vez que este tipo de colesterol não é prejudicial ao organismo. Observa-se que os níveis de HDL estão diminuindo com a idade, como se a depressão dessa fração do colesterol fosse o início do processo de distúrbio de metabolismo lipídico na história natural da doença. Sabe-se que o HDL tem função protetora, evitando a formação dos ateromas, num estágio inicial da doença aterosclerótica. O estudo prospectivo de Framingham ${ }^{22}$ mostrou que o risco de se adquirir doenças cardiovasculares ateroscleróticas é maior em indivíduos com baixas taxas de HDL, mesmo naqueles com níveis baixos de CT.

Apesar da redução nos níveis de CT e TG, alguns pacientes ainda apresentaram perfil lipídico alterado, em função de não terem recebido tratamento farmacológico. Tais alterações podem agravar a doença CV desses pacientes. Segundo o Programa Nacional de Educação sobre Colesterol $(\mathrm{NCEP})^{24}$ dos Estados Unidos, que reconhece as mudanças nos níveis plasmáticos de lipídios da população geral como um dos marcadores de risco tradicionais para aterosclerose coronariana, a doença cardiovascular aterosclerótica constitui um dos problemas mais sérios de saúde pública em muitos países, como o Brasil, ${ }^{25}$ porque muitos sujeitos com distúrbios lipídicos não são identificados ou são subtratados e, portanto, permanecem com um perfil lipídico desfavorável, aumentado, assim, o risco de eventos coronarianos. ${ }^{26}$

Todos os pacientes avaliados utilizavam dois ou mais medicamentos concomitantes. A prática de politerapia pela população idosa é elevada devido ao aumento de prevalência das doenças 
crônico-degenerativas nesta população. ${ }^{5}$ Os pacientes idosos são os principais consumidores e os maiores beneficiários da farmacoterapia moderna, mais de $80 \%$ tomam no mínimo um medicamento diariamente, sendo este o mais poderoso processo de intervenção para melhorar o estado de saúde dos mesmos. ${ }^{11,12}$

Considerando que o uso de medicamentos pelos idosos tem uma linha tênue entre o risco e o benefício, ou seja, a elevada utilização de medicamentos pode afetar a qualidade de vida do idoso, por outro lado, são os mesmos que, em sua maioria, ajudam a prolongar a vida. Logo, o problema não pode ser atribuído ao consumo do medicamento. ${ }^{27}$

Alguns medicamentos, quando administrados concomitantemente, podem causar interação medicamentosa, como no caso da sinvastatina e da genfibrozila, que quando administradas juntas, podem aumentar o risco de miopatia e rabdomiólise. Esta interação poderia resultar da interação dos fármacos com os transportadores hepáticos, levando à diminuição da captação das estatinas pelo fígado, ${ }^{28}$ sendo que a genfibrozila aumenta a área sob a curva da concentração sanguínea de todas as estatinas. ${ }^{29}$ Esta associação, no entanto, é utilizada principalmente em pacientes de alto risco e portadores de dislipidemia combinada e síndrome metabólica. ${ }^{30}$

Segundo a revisão dos dados da Veterans Administration Database, onde foram avaliados 93.677 pacientes que utilizavam genfibrozila combinada à estatina, não houve nenhum caso de rabdomiólise entre os pacientes. ${ }^{31}$ Sendo assim, a rabdomiólise é incomum, mas é o efeito adverso mais sério observado na terapia hipolipemiante com estatinas, ${ }^{32}$ porém não impede a associação de ambas, pois é um efeito raro relatado pelos pacientes.

O número de pacientes que relatou apresentar efeitos adversos com o uso de medicamentos antilipêmicos foi baixo, devido ao fato de os pacientes receberem acompanhamento durante todo o tratamento farmacológico, bem como cuidados por parte da equipe de enfermagem, diminuindo os efeitos adversos provocados pelos medicamentos. Assim, para garantir essa segurança aos pacientes, a equipe realiza pesquisas na literatura a fim de buscar mais informações sobre os medicamentos utilizados e, assim, relatar todos os possíveis efeitos adversos que poderão vir a ocorrer buscando evitá-los, consequentemente melhorando a qualidade de vida dos mesmos. O envelhecimento, porém, acarreta maior risco de desenvolvimento de reações adversas por parte dos idosos, sendo que estas são responsáveis por $10 \%$ a $20 \%$ das admissões hospitalares agudas. ${ }^{33}$ Assim, a avaliação do perfil lipídico, bem como a monitorização do uso de medicamentos, é de extrema importância, pois tem total relação com doenças cardiovasculares e suas implicações. ${ }^{5}$

Apesar de nem todas as intervenções farmacêuticas terem sido aceitas, o perfil lipídico desses pacientes melhorou após um ano de acompanhamento. As intervenções farmacêuticas permitiram a identificação de potenciais problemas relacionados aos medicamentos e a prevenção da grande maioria deles. ${ }^{34}$ Aos pacientes que não faziam administração de nenhum medicamento para o tratamento de dislipidemia, depois de realizadas intervenções farmacêuticas, foi prescrito pelo médico assistente o uso de medicamentos hipolipemiantes. Em função disto, os pacientes apresentaram melhora no perfil lipídico. Em algumas situações, porém, o médico avaliou todo o histórico clínico do paciente e optou por não prescrever tratamento medicamentoso, o que pode ser considerado como uma não-aceitação da intervenção farmacêutica proposta.

As principais limitações deste estudo podem estar relacionadas à escolha da amostra por conveniência, ou seja, foram selecionados pacientes residentes de apenas um local e com uma determinada patologia. Outra provável limitação pode estar relacionada ao tamanho da amostra.

Com base no presente estudo, pode-se verificar que foi possível buscar soluções frente às alterações encontradas no início da pesquisa. Assim, o farmacêutico pode auxiliar, por meio das intervenções farmacêuticas, no uso, horários e troca dos medicamentos. Destaca-se também a 
importância da realização de exames laboratoriais de rotina na população idosa, pois por meio destes foi possível diagnosticar e, consequentemente, oferecer um tratamento adequado, contribuindo para a melhora na assistência a este tipo de paciente.

Assim, os resultados permitiram adotar medidas específicas para prevenção dos erros de medicação detectados. A análise dos resultados obtidos sugere que a intervenção farmacêutica é uma ferramenta eficaz na detecção e prevenção de efeitos adversos, bem como na promoção

\section{REFERÊNCIAS}

1. Vieira EP, Miranda EC, Calais LL, Carvalho LMA, Iório MCM, Borges ACLC. Proposta de acompanhamento em grupo para idosos protetizados. Rev Bras Otorrinolaringol 2007;73(6):752-8.

2. Carvalho JAM, Garcia RA. O envelhecimento da população brasileira: um enfoque demográfico. Cad Saúde Pública 2003;19(3):725-33.

3. Ferré RJ, Morelló-Castro G. Validation of the otoacoustic emissions in presbyacusis. Acta Otorrinolaringol Esp 2003;54(3):177-82.

4. Ramos LR. Epidemiologia do envelhecimento. In: Freitas EV. Tratado de Geriatria e Gerontologia. Rio de Janeiro: Guanabara Koogan; 2002. p. 72-8.

5. Engroff P, Araújo PL, Schroeter G, Faggiani FT, Morrone FB, Gomes I, et al. Efeitos dos medicamentos hipolipêmicos no perfil lipídico de população idosa de Porto Alegre, RS, Brasil. RBAC 2008;40(4):297-300.

6. Grillo LP, Crispim SP, Siebert AN, Andrade ATW, Rossi A, Campos IC. Perfil lipídico e obesidade em escolares de baixa renda. Rev Bras Epidemiol 2005;8(1):75-81.

7. Sepúlveda JM. Dislipidemia nos idosos. Jornal Tribuna do Norte. 2007. [acesso em 19 mar 2010]. Disponível em: http://tribunadonorte.com.br/noticia. php?id=60689.

8. Rocha CH, Oliveira APSO, Ferreira C, Faggiani FT, Schroeter G, Souza ACA, et al. Adesão à prescrição médica em idosos de Porto Alegre, RS. Ciênc Saúde Coletiva 2008;13:703-10.

9. Rozenfeld S. Prevalência, fatores associados e mau uso de medicamentos entre os idosos: uma revisão. Cad Saúde Pública 2003;19(3):717-24.

10. Flores LM, Mengue SS. Uso de medicamentos por idosos em região do sul do Brasil. Rev Saúde Pública 2005;39(6):924-9. da integração do Serviço de Farmácia à equipe multiprofissional, pacientes e seus familiares. ${ }^{34}$

\section{CONCLUSÃO}

Observou-se uma melhora no perfil lipídico dos cinquenta pacientes dislipidêmicos em acompanhamento farmacoterapêutico após um ano, o que ressalta a importância desse tipo de companhamento buscando proporcionar melhor qualidade de vida aos idosos.

11. Beyth RJ, Shorr RI. Epidemiology of adverse drug reactions in the elderly by drug class. Drugs Aging 1999;14(3):231-9.

12. Monane M, Matthias DM, Nagle BA, Kelly MA. Improving prescribing patterns for the elderly through an online drug utilization review intervention: a system linking the physician, pharmacist, and compute. JAMA 1998;280(14):1249-52.

13. Anatomical Therapeutic Chemical Classification. Simposium Terapeutic. [acesso em 08 jul 2010]. Disponível em: http://www.simposium.pt/ medicamentos-classificacao-atc-S-5330.

14. Friedewald WT, Levy RL, Fredrickson DS. Estimation of the concentration of low-density lipoprotein cholesterol in plasma, without use of the preparative ultracentrifuge. Clin Chem 1972;18(6):499-502.

15. Di Piro JT, Talbert RL, Yee GC, Matzke GR, Wells BG, Posey L, editors. Pharmacotherapy: A Pathophysiologic Approach. 6th Edition. New York: McGraw-Hill; 2005.

16. Consenso Brasileiro sobre Dislipidemias: detecção, avaliação e tratamento. Arq Bras Endocrinol Metab 1999;43(4):287-305.

17. Sposito AC, Caramelli B, Fonseca FAH, Bertolami MC, Afiune Neto A, Souza AD, et al. IV Diretriz brasileira sobre dislipidemias e prevenção da aterosclerose da Sociedade Brasileira de Cardiologia. Arq Bras Cardiol 2007;88(supl I):2-19.

18. Gotto AM Jr. Triglyceride as a risk factor for coronary artery disease. Am J Cardiol 1998;82(9A):22Q-5Q.

19. Windler E, Schöffauer M, Zyriax BC. The significance of low HDL-cholesterol levels in na ageing society at increased risk for cardiovascular disease. Diab Vasc Dis Res 2007;4(2):136-42. 
20. McKenney JM. Pharmacotherapy of dyslipidemia. Cardiovasc Drugs Ther 2001;15(5):413-22.

21. Davidson MH, Maki KC, Karp SK, Ingram KA. Management of hypercholesterolaemia in postmenopausal women. Drugs Aging 2002;19(3):169-78.

22. Catelli PW, Garrison RJ, Wilson PW, Abbott RD, Kalousdian S, Kannel WB. Incidence of coronary heart disease and lipoprotein cholesterol levels. The Framingham Study. JAMA 1986;256(20):2835-8.

23. Goodman LS, Gilman A. As bases farmacológicas da terapêutica. 10 ed. Rio de Janeiro: McGraw-Hill; 2003.

24. Executive Summary of the Third Report of the National Cholesterol Education Program (NCEP). Expert Panel on Detection, Evaluation, and Treatment of High Blood Cholesterol in Adults (Adult Treatment Panel III). JAMA 2001;285(19):2486-97.

25. Santos RD. III Brazilian Guidelines on Dyslipidemias and Guideline of Atherosclerosis Prevention from Atherosclerosis Department of Sociedade Brasileira de Cardiologia. Arq Bras Cardiol 2001;77(suppl 3):1-48.

26. Gotto AM Jr. Treating hypercholesterolemia: looking forward. Clin Cardiol 2003;26(suppl 1):121-8.

27. Andrade MA, Silva MVS, Freitas O. Assistência Farmacêutica como Estratégia para o Uso Racional de
Medicamentos em Idosos. Semina Ciênc Biol Saúde 2004;25(1):55-63.

28. Shitara Y, Sato H, Sugiyama Y. Evaluation of drugdrug interaction in the hepatobiliary and renal transport of drugs. Annu Rev Pharmacol Toxicol 2005;45:689-723.

29. Davidson MH, Toth PP. Comparative effects of lipid-lowering therapies. Prog Cardiovasc Dis 2004;47(2):73-104.

30. Schulz I. Tratamento das dislipidemias: como e quando indicar a combinação de medicamentos hipolipemiantes. Arq Bras Endocrinol Metab 2006;50(2):344-59.

31. Backman JT, Kyrklund C, Neuvonen M, Neuvonen PJ. Gemfibrozil greatly increases plasma concentrations of cerivastatin. Clin Pharmacol Ther 2002;72(6):685-91.

32. Magalhães MEC. Mecanismo de rabdomiólise com as estatinas. Arq Bras Cardiol 2005;85(suppl 5):42-4.

33. Teixeira JJV, Lefevre F. Drug prescription from the perspective of elderly patients. Rev Saúde Pública 2001;35(2):207-13.

34. Nunes PHC, Nominato JCS, Albuquerque EM, Silva LFN, Castro IRS, Castilho SR. Intervenção farmacêutica e prevenção de eventos adversos. Rev Bras Cienc Farm 2008;44(4):691-9. 